Review

\title{
Advances in Nanocarriers for Drug Delivery in Dental Therapies
}

\author{
Bhaumik Patel ${ }^{1,}{ }^{*}$, Yeshwanthi Kamineni ${ }^{2}$, Shashank Gorityala ${ }^{3}$, M Hima Bindu ${ }^{4}$
}

1. Product Development Department, Cure Pharmaceutical Corporation, Los Angeles, CA 90025, USA; E-Mail: Bhaumikp17@gmail.com

2. Talasila Super Speciality Dental Hospital, Visakhapatnam, Andhra Pradesh, 530026, India; E-Mail: kamineniyesh@gmail.com

3. Department of Bioanalytical Chemistry, Covance Laboratories, Madison, WI, 53704, USA; E-Mail: shashank.gorityala@labcorp.com

4. Department of Pharmaceutical Analysis, School of Pharmacy, Anurag Group of Institutions, Ghatkesar, 500088, Telangana, India; E-Mail: bindu.maroju@gmail.com

* Correspondence: Bhaumik Patel; E-Mail: Bhaumikp17@gmail.com

Academic Editor: Eugeniusz Sajewicz

Special Issue: Advanced Dental Materials

Recent Progress in Materials

2021, volume 3, issue 3

doi:10.21926/rpm.2103039
Received: August 19, 2021

Accepted: September 17, 2021

Published: September 28, 2021

\begin{abstract}
Dental caries are one of the major causes of oral degeneration diseases. In order to treat dental-related diseases, it is often challenging and expensive. Recent literature has reported many advances in designing delivery systems utilizing polymers and novel biomaterials. Polymers play an important role in designing many nanoformulations like liposomes, polymeric micelles, polymeric nanoparticles, carbon-based nanoparticles, nanohydroxyapatite, iron oxide, zirconia, silica, and silver nanoparticles were used to treat oral complications of the oral cavity including dental caries, periodontal disease, and oral cancer. The present review focus on the importance of novel biodegradable polymers like poly $(D, L-$ Lactide acid), PLLA (poly-L-lactide), PDLA (poly-D- lactide), PLGA that is poly (D, L Lactide-coglycolide), cellulose acetate phthalate, and Chitosan, etc used to design site-specific delivery and controlled drug delivery to treat dental diseases. Moreover, research development in this
\end{abstract}

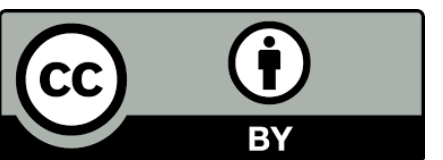

(C) 2021 by the author. This is an open access article distributed under the conditions of the Creative Commons by Attribution License, which permits unrestricted use, distribution, and reproduction in any medium or format, provided the original work is correctly cited. 
area will raise opportunities for the dentist, researchers, and pharmaceutical scientists to fabricate an ideal drug delivery system.

\section{Keywords}

Lipids; polymers; nanocarriers; drug delivery; dental; diseases

\section{Periodontal Diseases}

Perhaps the most harmful dental problem, influencing somewhere in the range of 5 to $30 \%$ of the adult population (25-75 years), is periodontitis [1]. Periodontal disease getting more far and wide in modern civilization and can be a premise for the beginning or worsening of diseases like respiratory diseases, diabetes, rheumatoid arthritis, malignancy, and metabolic syndrome. Periodontitis is a persistent inflammatory situation caused by the presence of bacterial plaque that may lead to the severe destruction of periodontal tissue. Even though periodontitis starts with the accumulation of bacteria, some other flattering factors can add to the provocation of the disease like (a) Local factors: Dental cavities, Smoking, tartar, and inappropriate nutrition, and (b) General factors: i) Incidence of few systemic diseases like anemia, leukemia, and diabetes, ii) Undergoing drug treatments for cardiovascular diseases or epilepsy and iii) Physiological conditions like menopause, pregnancy, and puberty.

In the beginning phases, the periodontal infection can be treated by less intrusive procedures like antibiotic treatment, root planning, or scaling. At a high-level stage, nonetheless, surgical treatment is utilized, including assisted tissue regeneration, soft tissue grafts, and flap surgery, etc. Periodontitis shows the advancement in the disease in three phases: Phase-1 Gingivitis; Phase IIModerate periodontitis and Phase III-Severe periodontitis. Periodontitis majorly affects few structures of the tooth like the marginal periodontium, dental cementum, and periodontal ligaments. Two major aspects of the treatment of periodontitis are, removal of affected biofilm and removal of bacterial species from the tooth surface [2]. Treponema denticola, Tannerella species, Porphyromonas gingivalis are the few microorganisms liable for triggering periodontitis [3].

Based on the standard treatment for periodontitis, the therapy involves two different phases depends upon the severity. The first phase includes displacement of dental plaque by scaling and root planning and the second phase consists of antibiotic therapy through oral administration [4]. While systemic antibiotic therapy has several shortcomings, there is a need to develop the most effective methods that can reduce bacterial adhesion and prevent biofilm formation.

This methodology can be acknowledged by utilizing the nanosystems along with an anti-microbial agent that can be used in the periodontal pocket as local drug delivery. The accomplishment of treating the periodontal infection with novel delivery systems will rely on the effectiveness of the system to deliver the agent at a proper concentration at the bottom of the periodontal pocket and retention of the system for prolonged periods [5]. In 1979, Goodson et al. projected the first controlled systems and so on many of the researchers continued their efforts to improve the current technologies for preciseness in the release as per the biological rhythms of the patient and disease condition. Local controlled drug systems can be classified as follows [6, 7]:

1. On basis of type of treatment application -Supra and subgingival irrigation 


\section{Following biodegradability of the device or system}

3. Depends on the type of device (Sustained or controlled)

An ideal dosage form to treat periodontitis is expected to be biodegradable, bioadhesive in nature along with sustained or controlled release over an extended time [8]. Chlorhexidine magnetic nanoparticles coated with amino silane showed a superior anti-fungal and bactericidal action against the deposited microbial film. These ideal nanoparticles are preferred owing to low toxicity against healthy human osteoblasts and improved antimicrobial activity in the presence of salivary proteins [9]. Another drug delivery system was fabricated for tetracyclines on basis of hydroxyapatite nanocarrier to eliminate the infection and promote bone regeneration. In vitro and in vivo studies confirmed that prepared nanoparticles were compatible and serve as bone substitutes along with anticipated antibacterial properties [10].

The liposomal-based drug delivery system was designed for doxycycline using $\mathrm{N}, \mathrm{N}, \mathrm{N}$ trimethyl chitosan showed exceptional biocompatibility, also prevented the formation of biofilm and bone resorption [11]. Another better way is to formulate nanoparticles by adding mineralizing agents like calcium phosphate along with the microbial agent, which can be further coated by carboxymethylcellulose to improve the bioadhesion. Subsequently, nanoparticles will bind to enamel as well as dentin to inhibit the growth of Lactobacillus cases and other bacteria [12, 13]. Nanoparticles can be easily administered into the periodontal pocket by injection. These systems can be applied very easily in a more accurate manner, which in turn reduces the cost of therapy in contrast with the other devices. Injectable nanoformulation for poly(dopamine) to treat oxidative stress-induced peridontitis, owing to its antioxidant efficiency in removing reactive oxygen species was reported. In-vitro and in-vivo evaluation studies revealed that the formulation showed admirable anti-inflammatory, ROS removal with good compatibility and low systemic toxicity.

Nanotechnology is shown to bridge the barrier of biological and physical sciences by applying nanostructures and nanophases at various fields of science; especially in nanomedicine and nanobased drug delivery systems, where such particles are of major interest. Nanomaterials can be welldefined as material with sizes ranging between 1 and $100 \mathrm{~nm}$, which influences the frontiers of nanomedicine starting from biosensors, microfluidics, drug delivery, and microarray tests to tissue engineering. Nanotechnology employs curative agents at the nanoscale level to develop nanomedicines. Nanomedicines have become well appreciated in recent times because nanostructures could be utilized as delivery agents by encapsulating drugs or attaching therapeutic drugs and deliver them to target tissues more precisely with a controlled release. Nanomedicine is an emerging field implementing the use of knowledge and techniques of nanoscience in medical biology and disease prevention and remediation. It implicates the utilization of nanodimensional materials for diagnosis, delivery, and sensory purposes, and actuate materials in live cells. For example, a nanoparticle-based method has been developed which combined both the treatment and imaging modalities of dental diagnosis as well [14]

Novel polymers play a crucial role in the design of nanoparticles. Few examples of Nanoparticles are fabricated by utilizing the polymers are poly (D, L-lactide corrosive) (PLA), poly(glycolic corrosive), poly(D, L-lactide-co-glycolide corrosive) (PLGA), and cellulose phthalate. Triclosan (TCS), which is anion cationic antimicrobial agent nanoparticles were prepared by the emulsification-diffusion technique by using cellulose phthalate. These TCS nanoparticles were infused into the lower part of periodontal pockets gradually decreases inflammation at the site of infection was seen after 15 days [15]. Quintanar-Guerrero and associates [16] prepared nanospheres and nanocapsules by using 
cellulose acetate phthalate as a pH-based polymer and loaded the chlorhexidine by the emulsiondiffusion method, for the treatment of periodontitis. Nanocapsules and nanospheres were injected into a periodontal pocket, and $65.78 \%$ less dental plaque was recorded. The formulation was then compared with marketed mouthwash shows that it is more effective. Due to their large surface area, smaller particle size, controlled release of medications at various $\mathrm{pH}$ values, shorter treatment time and dose, and potential to penetrate gingival tissues, these nanoparticle formulations are beneficial to dental use.

As examples of nanocarriers used in dental drug delivery, Chitosan, which is a natural polymer with various properties, can be utilized in the formulation of smart nanoparticles for the treatment of periodontal diseases as follows:

1. Chitosan crosslinked nanoparticles prepared by the emulsion-scattering technique loaded with sodium fluoride and cetylpyridinium chloride blended in with toothpaste lixivium to get a product that delivers the drug substance in a sustained manner [17];

2. To get protection against dental caries Chitosan crosslinked nanoparticles are formulated by ionic gelation method loaded with sodium fluoride used as dental drug delivery system [18];

3. For local drug action Chitosan-oligonucleotide complexes are often used.

4. Rose bengal- prepared smart chitosan nanoparticles having an affinity towards bacterial cells and helps in their elimination.

5. Lee and associates [19] prepared the potential core-shell poly(D, L-lactide-co glycolide)chitosan nanospheres loaded simvastatin and doxycycline for the treatment of periodontal diseases and found enormous benefits. and observed drug release for 28 days.

6. Chen and associates [20] formulated novel pH-responsive quaternary ammonium chitosanliposome nanoparticles for periodontal treatment. In-vivo tests showed that $\mathrm{pH}$-responsive nanoparticles act to inhibit biofilm formation and also helped in alveolar bone resorption, as well as excellent biocompatibility with human periodontal ligament fibroblasts.

7. Moreover, the pH-responsive nanoparticles can be utilized as a drug delivery system of doxycycline, which is an antibacterial agent used in the treatment of periodontal diseases.

8. Drugs, metronidazole (an antibiotic), and N-phenacylthiazolium bromide (a host modulator) nanospheres were prepared by encapsulating PLGA along with chitosan as an inflammationresponsive system by using oil in water single emulsion system using solvent evaporation strategy. These nanospheres administered into the subgingival portion shows reduced inflammation in the case of periodontitis [21].

\section{Dental Caries}

Dental caries is perhaps the most well-known and common dental infection [22]. The First grouping of dental caries used for a long time was acknowledged by G.V. black (1908) and at first composed of five classifications (Class 1-5), with a 6th being added later (Class 6, Simon's adjustment) [23]. Today, in light of the information about dental caries accumulated over the long run, much more elaborate characterizations have been created by a few standards [24]:

1. Anatomical site: Occlusal; proximal and cervical; straight veneer; and root caries;

2. Type of lesion: Primary and secondary caries.

3. Severity of the disease: Acute; chronic; and arrested caries.

4. Extent of caries: Incipient caries; occult caries; and cavitation. 
5. Tissues involved: Initial; superficial; moderate; deep; and deep complicate caries.

6. Pathway of caries spread: Forward and backward caries.

7. Number of tooth surfaces involved: Simple; compound; and complex caries.

8. Chronology: Early childhood; adolescent; and adult caries.

The main reasons for tooth decay are dental plaque development and the demineralization of teeth. To solve this problem, antibacterial and remineralization frameworks were created. Another method of preventing the generation of tooth decay is to develop DNA vaccines against $S$. mutans [25]. Yang and associates [26] developed a new strategy to deliver anti-caries DNA vaccine pGJA$\mathrm{P} /$ Vax into nasal mucosa in a $\mathrm{pH}$-mediated way is to develop a nanoparticle system by incorporating anionic liposomes into chitosan/DNA buildings can deliver the anti-caries. It was seen that the nanoparticles show higher transfection effectiveness, longer home time, more elevated level of secretory IgA, longer-term mucosal resistance, and negligible cytotoxicity, and supported clearance through the digestive tract, showed better properties compared with chitosan-DNA complexes. Smart nanoparticles of amorphous calcium phosphate were formulated by a spray drying procedure showed increases calcium and phosphate ions at a low $\mathrm{pH}$, which is important for minimize caries development, and also neutralize the lactic acid solution $(\mathrm{pH}=4)$ by increasing the $\mathrm{pH}$ to 6 , thereby avoid the formation of caries [27]. In addition, carbonate hydroxyapatite nanoparticles are an acceptable possibility for biomineralization and repair of sizable deformities on the tooth surface [28].

\section{Antiviral Delivery}

Viral infections are treated by antiviral drugs [29]. Although the majority of these agents are intended to target specific viruses, it is still possible to discover drugs that are effective against a wide range of viruses [30]. Viral infections are present on the skin of the mouth as well as the oral mucosa, one of the examples is oral herpes infection. Oral herpes is caused by the human herpes simplex virus 1 (HHV-1) which creates pain on the lips, tongue, and roof of the mouth.[31] Examples of other human herpes infections include the HHV-2 virus that causes genital herpes, the HHV-3 virus that causes chickenpox and herpes zoster, the HHV-4 (Epstein-Barr) and HSV-5 (cytomegalovirus) viruses that cause infectious mononucleosis, the HHV-6 and HHV-7 viruses that cause roseola (a viral disease causing high fever and a skin rash in small children), and the HHV-8 virus that causes Kaposi's sarcoma in people with acquired immune deficiency syndrome [32]. Systemic administration of antiviral drugs is the most viable method for treating oral virus infections.[33] In very rare cases local administration methods found in the literature and topical administration of antiviral agents creams for labial herpes is the only class of local drug delivery strategy available commercially.[34] To overcome the use of delivery of antiviral drugs to a specific site, the preparation of nanoparticles is an exciting field of research.[35] Nanoparticles can change the release kinetics of antiviral drugs, increases their bioavailability, control their dissolution rates, minimize their side effects, and lower the cost of treatment. nanoparticle delivery provides the possibility of targeting specific biological sites either passively or actively in dental diseases [36].

\section{Oral Mucositis}

One of the most common side effects during head and neck cancer treatment was found in oral mucositis [37]. A common oral disorder is a mucositis seen in $80 \%$ of patients undergoing 
radiotherapy and in $100 \%$ of patients receiving high-dose chemotherapy [38]. This condition is characterized by pain and discomfort, susceptibility to fungal and bacterial infections, and in the worst cases, sepsis. $4 \%$ rebamipide solution is usually prescribed by doctors to patients undergoing head and neck cancer therapy to prevent possible complications. However, its greatest weakness is that it may only remain in the oral cavity for a short time and therefore reduce drug effectiveness. To overcome this obstacle, chitosan-coated poly(lactide-co-glycolide) nanoparticles have been synthesized as carriers for rebamipide. One of the major characteristics of these nanoparticles was their positive surface charge, with a volume diameter of $100 \mathrm{~nm}$. These nanoparticles were coated with chitosan and were able to remain in the oral cavity for more than 6 hours as a result of their electrostatic interaction with the negatively-charged carboxyl groups of mucin within the oral mucosa. In comparison with rebamipide suspension alone, chitosan-coated nanoparticles demonstrated superior results and shorter treatment times [39]. Similarly, promising results were achieved with gold nanoparticles on hamsters when used in treating induced oral mucositis [40]. In general, oral ulcers (also known as canker sores or recurrent aphthous stomatitis) are painful lesions that affect the mouth. They are often caused by infections, immune disorders, trauma, or cancer [41]. In most cases, oral ulcers are treated by reducing or eliminating pain and preventing secondary infections, depending on the underlying cause. Radiation-induced oral ulcers in mice were significantly improved with Aloe vera solutions combined with silver nanoparticles [42]. Successful treatment of the condition with oral mucoadhesive films containing propolis extract was demonstrated in human clinical trials. Through this drug delivery system, the pain was reduced for a longer period and healing was enhanced [43].

\section{Prosthodontics and Orthodontics}

The field of prosthodontics is critical for replacing lost teeth as well as restoring facial appearance and function. An option for treatment is to use removable partial or full dentures or to use fixed tooth support or implant prosthetics. Recently, nanoparticles are becoming prevalent in prosthodontics, are used in ceramics, resins, and metals [44]. A common denture base material, polymethyl methacrylate (PMMA) is used because of its biocompatibility, lightweight, low cost, and stability. PMMA-based dentures, however, tend to become colonized easily by bacterial and fungal biofilms and are prone to fractures [45]. The drawbacks of PMMA have been overcome by modifying it in numerous ways. The surface hydrophobicity of PMMA-based dentures has been enhanced with nanoparticles to provide antimicrobial activity. A denture base acrylic resin containing silver nanoparticles was reported and it did not affect the adhesion of $C$. alphabicans and biofilm formation [46]. However, it has been shown that silver nanoparticles had antifungal activity and reduced C.albicans formation [47, 48]. The incorporation of poly(diallyldimethylammonium) chloride nanoparticles into PMMA produced an antibacterial effect against $E$. coli, S. aureus, and $C$. albicans [49]. Similarly, PMMA denture acrylic containing platinum nanoparticles had a significant bacterial anti-adherent effect [50]. Improving the antibacterial capacity of fixed orthodontics appliances has been crucial in dentistry to prevent the development of white spot lesions, which is the most common side effect associated with the placement of orthodontic brackets. An orthodontic adhesive incorporating curcumin-doped poly(lactic-co-glycolic acid) nanoparticles were evaluated for its anti-biofilm efficacy against S. mutans biofilms. The effectiveness of the drug- 
loaded nanocarriers was confirmed and may be used as an antibacterial and antibiofilm orthodontic adhesive [51].

\section{Anti-Infective Nanofibers and Other Applications in Dentistry}

Despite their popularity, dental implants are susceptible to biofilm formation, leading to periimplantitis and eventual implant failure [52]. There has been researched to develop implants that have an osteointegration surface while reducing the formation of biofilms [53]. Nanofibers containing PCL/tetracycline $(5,10$, and $25 \% \mathrm{wt})$ were tested for their antimicrobial effects against periimplantitis-associated microorganisms such as $P$. gingivalis, $F$. nucleatum, $P$. intermedia, and actinobacillus actinomycetemcomitans. Nanofibers incorporating $25 \%$ wt of tetracycline were able to inhibit $100 \%$ of the biofilms of these bacteria [54]. Nanomaterials could be used to treat implant surfaces in the future. Another common problem in dentistry is fungal infections [55]. Most of these infections are caused by $C$. albicans mainly affects patients with full dentures, patients with some systemic condition that reduces their immune response, or even patients who use antibiotics that reduce their commensal oral microbiota [56]. Therefore, nanofibers could be used as a delivery method for antifungals [22]. Nanofibers containing polyvinylpyrrolidone (PVP) and poly (methyl methacrylate) (PMMA), incorporated with cetylpyridinium chloride (CPC) (5\% wt) or miconazole (5\% wt) showed in-vitro antimicrobial activity through zones of inhibition against $C$. albicans after $48 \mathrm{~h}$ of treatment.

A new study was done by Tonglairoum and colleagues [57] that developed clotrimazole nanofibers composed of several polymers, including polyvinyl pyrollidone (PVP), Chitosan (CS), and polyvinyl alcohol (PVA). They observed that clotrimazole was released ( $80 \%$ ) in saliva and buffer solution within $4 \mathrm{~h}$ and these nanofibers were able to completely inhibit the Growth of C.albicans after 3 hours of incubation. In addition, these nanofibers did not reduce the viability of human gingival fibroblasts after $24 \mathrm{~h}$ of incubation [58]. Therefore, there is a barrier between the application of nanofibers in dental implants and prosthetics. Moreover, these nanofibers were not able to reduce the cell viability of human gingival fibroblasts after $24 \mathrm{~h}$ of incubation [59]. Therefore, there is still a barrier between in-vitro and clinical studies regarding the application of nanofibers both in dental implants and prostheses. Moreover, these nanofibers were not able to reduce the cell viability of human gingival fibroblasts after $24 \mathrm{~h}$ of incubation [60-66]. Therefore, there is still a barrier between in-vitro and clinical studies regarding the application of nanofibers both in dental implants and prostheses. However, all these new possibilities can contribute to the development of implants with antimicrobial characteristics, or antifungal mucoadhesive nanofibers to be coupled between the prosthesis and the oral mucosa. Therefore, there is still a barrier between in-vitro and clinical studies regarding the application of nanofibers both in dental implants and prostheses. The new possibilities can, however, lead to the development of implants with antimicrobial properties, or antifungal mucoadhesive nanofibers coupled between the prosthesis and the oral mucosa.

\section{Conclusion}

Among the various diseases that secure a place in the oral cavity in combination with the difficulty of reaching an effective concentration of the drug at the diseased area, for sure there is a demand for novel drug delivery nanocarriers in the localized oral therapies. Most of the nanocarriers have not reached the market yet since it involves in great scale-up challenges. There are many 
advances in both cell- and gene-delivery-based nanotechnologies and their combined use of nanoparticulate formulations as promising modalities in dental therapies. There is still a broad scope of improvement in existing conventional and novel nanocarriers for the treatment of dental diseases. The other major concerns with nanotechnology-based carries are toxicity, safety, tissue properties, and their potential internalization, these aspects need a very close look. Better infrastructure and scale-up modalities are enabling to overcome such limitations and yielding to move more products into the market.

\section{Author Contributions}

Bhaumik Patel: Project administration, resources, investigation, formal analysis, writing review \& editing. Yeshwanthi Kamineni: Data curation, investigation, methodology, writing- review \& editing. Shashank Gorityala: Investigation, methodology, resources, writing- review \& editing. Hima Bindu: Formal Analysis, methodology, writing original -draft, Formal analysis.

\section{Competing Interests}

The authors have declared that no competing interests exist.

\section{References}

1. Bhardwaj A, Bhardwaj SV. Local drug delivery in periodontology. Recent Adv Pharm Res. 2012; 1: 1-5.

2. Liang J, Peng X, Zhou X, Zou J, Cheng L. Emerging applications of drug delivery systems in oral infectious diseases prevention and treatment. Molecules. 2020; 25: 516.

3. Alsuraifi A. Metallic nanoparticles in dental biomaterials: A review. AAJMS. 2020; 3: 27-37.

4. Ahuja A, Rahman S, Ali J, Khar RK. Site-specific delivery system for treatment of periodontitis. Indian J Pharm Sci. 2003; 65: 106-112.

5. Kaplish V, Walia MK, Hari Kumar SL. Local drug delivery systems in the treatment of periodontitis: A review. Pharmacophore. 2013; 4: 39-49.

6. Dodwad V, Vaish S, Chhoka M, Mahajan A. Magic bullet to treat periodontitits: A targeted approach. J Pharm Biomed Sci. 2012; 20: 1-5.

7. Rams TE, Slots J. Local delivery of antimicrobial agents in the periodontal pocket. Periodontol 2000. 1996; 10: 139-159.

8. Vyas SP, Shihorkar V, Mishra V. Controlled and targeted drug delivery strategies towards intraperiodontal pocket diseases. J Clin Pharm Therapeut. 2000; 25: 21-42.

9. Tokajuk G, Niemirowicz K, Deptula P, Piktel E, Ciesluk M, Wileczewska AZ, et al. Use of magnetic nanoparticles as a drug delivery system to improve chlorhexidine antimicrobial activity. Int J Nanomed. 2017; 12: 7833-7846.

10. Madhumathi K, Sampath Kumar TS. Regenerative potential and antibacterial activity of tetracycline loaded apatitic nanocarriers for the treatment of periodontitis. Biomed Mater. 2014; 9: 035002.

11. Kurakula M, Naveen NR, Patel B, Manne R, Patel DB. Preparation, optimization and evaluation of chitosan-based avanafil nanocomplex utilizing antioxidants for enhanced neuroprotective effect on PC12 cells. Gels. 2021; 7: 96. 
12. Kovtun A, Kozlova D, Ganesan K, Biewald C, Seipold N, Gaengler P, et al. Chlorhexidine-loaded calcium phosphate nanoparticles for dental maintenance treatment combination of mineralizing and antibacterial effect. RCS Adv. 2012; 2: 870-875.

13. Bao X, Zhao J, Sun J, Hu M, Yang X. Polydopamine nanoparticles as efficient scavengers for reactive oxygen species in periodontal disease. ACS Nano. 2018; 12: 8882-8892.

14. Vidal-Romero G, Zambrano-Zaragoza ML, Martinez-Acevedo L, Leyva-Gomez G, Mendoza-Elvira $\mathrm{SE}$, Quintanar-Guerrero D. Design and evaluation of $\mathrm{pH}$-dependent nanosystems based on cellulose acetate phthalate, nanoparticles loaded with chlorhexidine for periodontal treatment. Pharmaceutics. 2019; 11: 604.

15. Liu H, Chen B, Mao Z, Gao C. Chitosan nanoparticles for loading of tooth paste actives and adhesion on tooth analogs. J Appl Polym Sci. 2007; 106: 4248-4256.

16. Nguyen S, Escudero C, Seidiqi N, Smistad G, Hiorth M. Fluoride loaded polymeric nanoparticles for dental delivery. Eur J Pharm Sci. 2017; 104: 326-334.

17. Dung TH, Lee SR, Han SD, Kim SJ, Ju YM, Kim MS, et al. Chitosan TPP nanoparticles as a release system of antisense oligonucleotide in the oral environment. J Nanosci Nanotechnol. 2007; 11: 3695-3699.

18. Shrestha A, Kirshen A. Antibacterial efficacy of photosensitizer functionalized biopolymeric nanoparticles in the presence of tissue inhibitors in root canal. J Endod. 2014; 40: 566-570.

19. Chang PC, Tai WC, Luo HT, Lai CH, Lin HH, Lin ZJ, et al. Core-shell poly-(D, L-Lactide-co-Glycolide)chitosan nanospheres with simvastatin-doxycycline for periodontal and osseous repair. Int J Biol Macromol. 2020; 158: 627-635.

20. Hu F, Zhou Z, Xu Q, Fan C, Wang L, Ren H, et al. A novel pH-responsive quaternary ammonium chitosan-liposome nanoparticles for periodontal treatment. Int J Biol Macromol. 2019; 129: 1113-1119.

21. Patel DB, Dave RH. Predicting lubricants effect on tablet sticking using ketoprofen as model drug and evaluating sticking propensity using different metals and powder rheology. Int J Pharm. 2021; 606: 120913.

22. Lin JH, Feng F, Yu MC, Wang $\mathrm{CH}$, Chang PC. Modulation of periodontitis progression using $\mathrm{pH}$ responsive nanosphere encapsulating metronidazole or $\mathrm{N}$-phenacylthiazolium bromide. J Periodont Res. 2018; 53: 22-28.

23. Ahmadian E, Shahi S, Yazdani J, Dizaj SM, Sharifi S. Local treatment of the dental caries using nanomaterials. Biomed Pharmacother. 2018; 108: 443-447.

24. Kurakula M, Gorityala S, Patel DB, Basim P, Patel B, Kumar Jha S. Trends of chitosan based delivery systems in neuroregeneration and functional recovery in spinal cord injuries. Polysaccharides. 2021; 2: 519-537.

25. Fisher J, Glick M. A new model for caries classification and management: The FDI World Dental Federation caries matrix. J Am Dent Assoc. 2012; 143: 546-551.

26. Chen L, Zhu J, Li Y, Lu J, Gao L, Xu H, et al. Enhanced nasal mucosal delivery and immunogenicity of anticaries DNA vaccine through incorporation of anionic liposomes in chitosan/DNA complexes. PLoS ONE. 2013; 8: e71953.

27. Cheng L, Zhang K, Weir MD, Melo MA, Zhou X, Xu HH. Nanotechnology strategies for antibacterial and remineralizing composites and adhesives to tackle dental caries. Nanomedicine. 2015; 10: 627-641. 
28. Roveri N, Palazzo B, lafisco M. The role of biomimetism in developing nanostructured inorganic matrices for drug delivery. Expert Opin Drug Deliv. 2008; 5: 861-887.

29. Moosa MY, Sobel JD, Elhalis H, Du W, Akins RA. Fungicidal activity of fluconazole against candida albicans in a synthetic vagina-simulative medium. Antimicrob Agents Chemother. 2004; 48: 161-167.

30. Rençber S, Karavana SY, Yılmaz FF, Eraç B, Nenni M, Özbal S, et al. Development, characterization, and in vivo assessment of mucoadhesive nanoparticles containing fluconazole for the local treatment of oral candidiasis. Int J Nanomedicine. 2016; 11: 2641-2653.

31. Liu M, Zhang J, Zhu X, Shan W, Li L, Zhong J, et al. Efficient mucus permeation and tight junction opening by dissociable "mucus-inert" agent coated trimethyl chitosan nanoparticles for oral insulin delivery. J Control Release. 2016; 222: 67-77.

32. Dhoke DM, Basaiyye SS, Khedekar PB. Development and characterization of L-HSA conjugated PLGA nanoparticle for hepatocyte targeted delivery of antiviral drug. J Drug Delivery Sci Technol. 2018; 47: 77-94.

33. Chen L, Liang J. An overview of functional nanoparticles as novel emerging antiviral therapeutic agents. Mater Sci Eng C. 2020; 112: 110924.

34. Lotufo MA, Tempestini Horliana AC, Santana T, de Queiroz AC, Gomes AO, Motta LJ, et al. Efficacy of photodynamic therapy on the treatment of herpes labialis: A systematic review. Photodiagn Photodyn Ther. 2020; 29: 101536.

35. Mishra E, Goyal AK, Rath G. Recent advances in drug delivery systems in antiviral therapy. Pharmaspire. 2018; 10: 98-106.

36. Delbem AC, Pessan JP. Fluoride agents and dental caries. In: Pediatric restorative dentistry. Cham, Switzerland: Springer, Cham; 2019.

37. Harris DJ. Cancer treatment-induced mucositis pain: Strategies for assessment and management. Ther Clin Risk Manage. 2006; 2: 251-258.

38. Rubenstein EB, Peterson DE, Schubert M, Keefe D, McGuire D, Epstein J, et al. Clinical practice guidelines for the prevention and treatment of cancer therapy-induced oral and gastrointestinal mucositis. Cancer. 2004; 100: 2026-2046.

39. Takeuchi I, Kamiki Y, Makino K. Therapeutic efficacy of rebamipide-loaded PLGA nanoparticles coated with chitosan in a mouse model for oral mucositis induced by cancer chemotherapy. Colloids Surf B Biointerfaces. 2018: 167; 468-473.

40. Ribeiro SB, de Araújo AA, Oliveira MM, Santos Silva AM, da Silva-Júnior AA, Guerra GC, et al. Effect of dexamethasone-loaded PLGA nanoparticles on oral mucositis induced by 5fluorouracil. Pharmaceutics. 2021; 13: 53.

41. Vilar CJ, Ribeiro SB, de Araujo AA, Guerra GC, de Araujo Junior RF, Brito GA, et al. Effect of Gold nanoparticle on 5 -fluorouracil-induced experimental oral mucositis in hamsters. Pharmaceutics. 2020; 12: 304.

42. Fitzpatrick SG, Cohen DM, Clark AN. Ulcerated lesions of the oral mucosa: Clinical and histologic review. Head Neck Pathol. 2019; 13: 91-102.

43. El-Batal Al, Ahmed SF. Therapeutic effect of Aloe vera and silver nanoparticles on acid-induced oral ulcer in gamma-irradiated mice. Braz Oral Res. 2018; 32: e004.

44. Arafa MG, Ghalwash D, El-Kersh DM, Elmazar MM. Propolis-based niosomes as oromucoadhesive films: A randomized clinical trial of a therapeutic drug delivery platform for the treatment of oral recurrent aphthous ulcers. Sci Rep. 2018; 8: 18056. 
45. Priyadarsini S, Mukherjee S, Mishra M. Nanoparticles used in dentistry: A review. J Oral Biol Craniofacial Res. 2017; 8: 58-67.

46. Gad MM, Abualsaud R, Al-Thobity AM, Baba NZ, Al-Harbi FA. Influence of addition of different nanoparticles on the surface properties of poly(methylmethacrylate) denture base material. J Prosthodontics. 2020; 29: 422-428.

47. Wady AF, Machado AL, Zucolotto V, Zamperini CA, Berni E, Vergani CE. Evaluation of Candida albicans adhesion and biofilm formation on a denture base acrylic resin containing silver nanoparticles. J Appl Microbiol. 2012; 112: 1163-1172.

48. Patel DB, Tiyyagura P, Patel B. Trends in Nanocarrier based Delivery Systems of Methotrexate: Update 2020. Cancer Sci Res. 2021; 4: 1-7.

49. Nascimento C, Sanches LM, Mathiazzi BI, Ribeiro RT, Freitas D, Petri S, et al. Antimicrobial coatings from hybrid nanoparticles of biocompatible and antimicrobial polymers. Int J Mol Sci. 2018; 19: 2965.

50. Nam K. Characterization and bacterial anti-adherent effect on modified PMMA denture acrylic resin containing platinum nanoparticles. J Adv Prosthodont. 2014; 6: 207-214.

51. Ahmadi H, Haddadi-AsI V, Ghafari HA, Ghorbanzadeh R, Mazlum Y, Bahador A. Shear bond strength, adhesive remnant index, and anti-biofilm effects of a photoexcited modified orthodontic adhesive containing curcumin doped poly lactic-co-glycolic acid nanoparticles: An ex-vivo biofilm model of S. mutans on the enamel slab bonded brackets. Photodiagn Photodyn Ther. 2020; 30: 101674.

52. Shahi RG, Albuquerque MT, Münchow EA, Blanchard SB, Gregory RL, Bottino MC. Novel bioactive tetracycline-containing electrospun polymer fibers as a potential antibacterial dental implant coating. Odontology. 2017; 105: 353-363.

53. Carcuac O, Derks J, Abrahamsson I, Wennström JL, Petzold M, Berglundh T. Surgical treatment of peri-implantitis: 3-year results from a randomized controlled clinical trial. J Clin Periodontol. 2017; 44: 1294-1303.

54. Wang $M$, Tang T. Surface treatment strategies to combat implant-related infection from the beginning. J Orthop Transl. 2019; 17: 42-54.

55. Telles DR, Karki N, Marshall MW. Oral fungal infections: Diagnosis and management. Dent Clin North Am. 2017; 61: 319-349.

56. Kurakula M, Patel DB, Patel B, Gorityala S, Basim P. Functionalized Nanocarriers for Drug delivery: Amalgam of Biopolymers and Lipids. J Nanomed. 2021; 4: 1037.

57. Tonglairoum $P$, Ngawhirunpat $T$, Rojanarata $T$, Panomsuk S, Kaomongkolgit R, Opanasopit $P$. Fabrication of mucoadhesive chitosan coated polyvinylpyrrolidone/cyclodextrin/clotrimazole sandwich patches for oral candidiasis. Carbohydr Polym. 2015; 132: 173-179.

58. Engelmann J, Leyhausen G, Leibfritz D, Geurtsen W. Effect of TEGDMA on the intracellular glutathione concentration of human gingival fibroblasts. J Biomed Mater Res. 2002; 63: 746751.

59. Moharamzadeh K, Van Noort R, Brook IM, Scutt AM. Cytotoxicity of resin monomers on human gingival fibroblasts and HaCaT keratinocytes. Dent Mater. 2007; 23: 40-44.

60. Ebrahimi N, Soleimani AA, Rashidiani J, Malekafzali B, Abedini F, Hosseinkhani H. Chitosan/fluoride nanoparticles for preventing dental caries. Curr Dent. 2019; 1. doi: $10.2174 / 2542579 \times 01666190212150457$. 
61. Hosseinkhani H, Domb AJ. Biodegradable polymers in gene-silencing technology. Polym Adv Technol. 2019; 30: 2647-2655.

62. Abedini F, Ebrahimi M, Roozbehani AH, Domb AJ, Hosseinkhani H. Overview on natural hydrophilic polysaccharide polymers in drug delivery. Polym Adv Technol. 2018; 29: 2564-2573.

63. Mottaghitalab F, Farokhi M, Shokrgozar MA, Atyabi F, Hosseinkhani H. Silk fibroin nanoparticle as a novel drug delivery system. J Control Release. 2015; 206: 161-176.

64. Mazzarino L, Borsali R, Lemos-Senna E. Mucoadhesive films containing chitosan - coated nanoparticles: A new strategy for buccal curcumin release. J Pharm Sci. 2014; 103: 3764-3771.

65. Clarkson E, Mashkoor F, Abdulateef S. Oral viral infections: Diagnosis and management. Dent Clin North Am. 2017; 61: 351-363.

66. Acosta-Torres LS, Mendieta I, Nuñez-Anita RE, CajeroJuárez M, Castaño VM. Cytocompatible antifungal acrylic resin containing silver nanoparticles for dentures. Int J Nanomed. 2012; 7: 4777-4786.

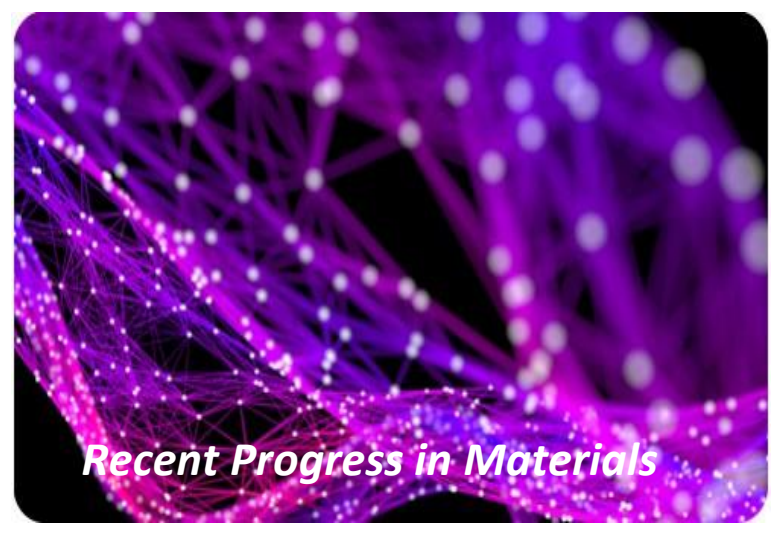

Enjoy Recent Progress in Materials by:

1. Submitting a manuscript

2. Joining in volunteer reviewer bank

3. Joining Editorial Board

4. Guest editing a special issue

For more details, please visit:

http://www.lidsen.com/journals/rpm 